\title{
Antioptimization of Earthquake Excitation and Response
}

\author{
G. ZUCCARO ${ }^{\text {a }}$, I. ELISHAKOFF ${ }^{\mathrm{b}, *}$ and A. BARATTA ${ }^{\mathrm{c}}$ \\ a Centro di Ricerca Interdipartimentale, LUPT, ${ }^{\circ}$ Dipartimento di Scienza delle \\ Costruzioni, University of Naples, Italy; ${ }^{b}$ Department of Mechanical Engineering, \\ Florida Atlantic University, Boca Raton, FL 33431-0991, USA
}

(Received 3 March 1995; In final form 14 July 1997)

The paper presents a novel approach to predict the response of earthquake-excited structures. The earthquake excitation is expanded in terms of series of deterministic functions. The coefficients of the series are represented as a point in $N$-dimensional space. Each available accelerogram at a certain site is then represented as a point in the above space, modeling the available fragmentary historical data. The minimum volume ellipsoid, containing all points, is constructed. The ellipsoidal models of uncertainty, pertinent to earthquake excitation, are developed. The maximum response of a structure, subjected to the earthquake excitation, within ellipsoidal modeling of the latter, is determined. This procedure of determining least favorable response was termed in the literature (Elishakoff, 1991) as an antioptimization. It appears that under inherent uncertainty of earthquake excitation, antioptimization analysis is a viable alternative to stochastic approach.

Keywords: Convex analysis; Ellipsoidal modeling; Earthquake engineering

\section{INTRODUCTION}

There is a vast literature on modeling of earthquake excitations. The modern analysis is based on the recognition that this excitation is an uncertain process. In overwhelming majority of the studies, the uncertainty is modeled as a random process, either stationary or non-stationary, with various approximations and attendant models.

\footnotetext{
* Corresponding author.
} 
The works by Drenick [1-4], Shinozuka [5], Elishakoff and Pletner [6], Baratta and Zuccaro [8,9] and have been the exceptions in the literature dedicated to uncertainty modeling of the earthquakes, in the sense that they utilized an alternative, non-probabilistic, avenue. Drenick $[1,2]$ used a constraint on the total energy which the earthquake is likely to develop at a certain site, as a description of uncertainty. He used the Cauchy-Schwarz inequality to determine the maximum response of the system to such an excitation. In the opinion of several investigators such a bound was too conservative [7]. Shinozuka [5] has suggested to characterize the earthquake uncertainty by specifying an envelope of the Fourier amplitude spectrum. Numerical calculations have demonstrated that the maximum response of the structure predicted by this method is less than that predicted in Refs. [1-4]. Elishakoff and Pletner [6] investigated the modification of the response prediction when the global information on the excitation is increased. In particular, the maximum possible response, which the structure may develop, was evaluated under the assumption that only the bound on base acceleration is known; then the maximum response was modified under the assumption that in addition to the base acceleration bound, the bounds on base velocity and/or displacement were specified. Baratta and Zuccaro in Refs. [8,9,17-19] developed a technique to produce the maximum theoretical values of the structural response under seismic load at given site. They pursued the goal combining available techniques for synthesis of random accelerograms (Ruiz-Penzien, 1971) with the optimization procedures capable of maximizing some parameters, significant for aseismic design, in respect of the constraints represented by the basic values characteristic of the shaking properties at the site.

In this paper we resort to ellipsoidal modeling of earthquake excitation. The ellipsoidal model of uncertainty has been previously introduced in the theory of control by Schweppe [10], and in modeling geometric imperfections in structures by Ben-Haim and Elishakoff [11] (see also an essay by Elishakoff [12]). Only other work known to us, on using convex modeling to earthquake engineering is that by Ben-Haim, Chen and Soong [13]. They suggested several analytical treatments, without resorting to the data analysis, that is the central topic of the present investigation. 


\section{FORMULATION OF THE PROBLEM}

Consider a single-degree-of-freedom linear system, subjected to earthquake excitation

$$
m \ddot{x}+c \dot{x}+k x=-m \ddot{x},
$$

where $x(t)=$ displacement of the structure, $c=$ damping coefficient, $k=$ stiffness, $\ddot{x}_{\mathrm{b}}(t)=$ earthquake excitation. If the excitation force $-m \ddot{x}_{\mathrm{b}}$ is known, the response is given by the Duhamel integral

$$
x(t)=\int_{0}^{t}\left[-m \ddot{x}_{\mathrm{b}}(\tau)\right] h(t-\tau) \mathrm{d} \tau,
$$

where $h(t)$ is the impulse response function

$$
\begin{aligned}
h(t) & =\frac{\exp \left(-\xi \omega_{0} t\right)}{m \omega_{\mathrm{d}}} \sin \omega_{\mathrm{d}} t, \\
\omega_{\mathrm{d}} & =\omega_{0} \sqrt{1-\xi^{2}},
\end{aligned}
$$

where $\omega_{0}=\sqrt{k / m}$ is the natural frequency, and $\xi=c / 2 \sqrt{k m}$ is the damping ratio. Let us represent the excitation as a series in terms of complete and orthogonal deterministic set of functions $\{\varphi(t)\}$, in the interval $(0, T)$ where $T$ is the duration of the earthquake

$$
\int_{0}^{T} \varphi_{i}(t) \varphi_{j}(t) \mathrm{d} t=0, \quad \text { for } i \neq j,
$$

namely,

$$
\ddot{x}_{\mathrm{b}}=\sum_{i=1}^{\infty} A_{i} \varphi_{i}(t) .
$$

If the excitation is known, the coefficients $A_{i}$, are readily obtainable, by multiplying both sides of Eq. (5) by $\varphi_{j}(t)$, and integrating the result in the interval $(0, T)$, and using the orthogonality property (5):

$$
A_{i}=\frac{1}{V_{i}^{2}} \int_{0}^{T} \ddot{x}_{\mathrm{b}}(t) \varphi_{i}(t) \mathrm{d} t,
$$


where

$$
V_{i}^{2}=\int_{0}^{T} \varphi_{i}^{2}(t) \mathrm{d} t
$$

The response, given in Eq. (2), is re-written as

$$
x(t)=\sum_{i=j}^{\infty} A_{i} \psi_{i}(t)
$$

where

$$
\psi_{i}(t)=-m \int_{0}^{T} \varphi_{i}(t) h(t-\tau) \delta \tau .
$$

Let us visualize now that at a given site we possess some fragmentary information, namely we assume that the historical data is available on the accelerograms $\ddot{x}_{\mathrm{b}}^{(1)}, \ddot{x}_{\mathrm{b}}^{(2)}, \ldots, \ddot{x}_{\mathrm{b}}^{(m)}$ where the superscript denotes the serial number of the earthquake, and $m$ is the total number of accelerograms. Then, using the decomposition of type (6) for each earthquake realization,

$$
x_{\mathrm{b}}^{(k)}=\sum_{i=1}^{\infty} A_{i}^{(k)} \varphi_{i}(t), \quad k=1,2, \ldots, m,
$$

we arrive at $m$ vectors of the excitation parameters

$$
\begin{aligned}
& \boldsymbol{A}^{(1) \mathrm{T}}=\left[\begin{array}{llll}
A_{1}^{(1)} & A_{2}^{(1)} & \ldots & A_{N}^{(1)}
\end{array}\right], \\
& \boldsymbol{A}^{(2) \mathrm{T}}=\left[\begin{array}{llll}
A_{1}^{(2)} & A_{2}^{(2)} & \ldots & A_{N}^{(2)}
\end{array}\right], \\
& \vdots \\
& \boldsymbol{A}^{(m) \mathrm{T}}=\left[\begin{array}{llll}
A_{1}^{(m)} & A_{2}^{(m)} & \ldots & A_{N}^{(m)}
\end{array}\right],
\end{aligned}
$$

where the superscript $\mathrm{T}$ means transposition. In addition we have retained only the most significant $N$ terms in the series (6)

$$
\ddot{x}_{\mathrm{b}}(t)=\sum_{i=1}^{N} A_{i} \varphi_{i}(t), \quad x(t)=\sum_{i=1}^{N} B_{i} \varphi_{i}(t) .
$$


Thus we replace $m$ accelerograms by $m$ points in an $N$-dimensional space. To this end, to use the ellipsoidal modeling of these "pointimages" of original accelerogram, we model these points as belonging to an $N$-dimensional ellipsoid

$$
\boldsymbol{A}^{\mathrm{T}} \boldsymbol{W} \boldsymbol{A} \leq \theta^{2},
$$

where $\boldsymbol{W}$ is a symmetric positive-definite matrix, defining the shape of the ellipsoid, $\theta^{2}$ is a positive constant, defining the size of the ellipsoid. The way of obtaining the matrix $\boldsymbol{W}$ and constant $\theta^{2}$ will be discussed in Section 4.

\section{MAXIMUM STRUCTURAL RESPONSE}

We are interested in determining the maximum response of the structure at any time instant $t$. Mathematically the problem reads as follows:

$$
\max \sum_{i=1}^{N} A_{i} \psi_{i}(t)=\max \boldsymbol{A}^{\mathrm{T}} \boldsymbol{\psi}(t), \quad \text { subject to } \boldsymbol{A}^{\mathrm{T}} \boldsymbol{W} \boldsymbol{A} \leq \theta^{2}
$$

The closely related problem has been studied in Refs. [11,12]. For the sake of completeness the basic steps of Ref. [12] will be re-introduced here, although in a different context. We define the Lagrangian

$$
L=\boldsymbol{A}^{\mathrm{T}} \boldsymbol{\psi}+\lambda\left(\boldsymbol{A}^{\mathrm{T}} \boldsymbol{W} \boldsymbol{A}-\theta^{2}\right),
$$

where the vector $\psi$ is defined as

$$
\boldsymbol{\psi}^{\mathrm{T}}=\left[\begin{array}{llll}
\psi_{1}(t) & \psi_{2}(t) & \ldots & \psi_{N}(t)
\end{array}\right] .
$$

The necessary conditions for the maximum reads

$$
0=\frac{\mathrm{d} L}{\mathrm{~d} \boldsymbol{A}}=\boldsymbol{\psi}+2 \lambda \boldsymbol{W} \boldsymbol{A} .
$$

We multiply from the left this equation by $\boldsymbol{W}^{-1}$ :

$$
\boldsymbol{A}=\frac{-1}{2 \lambda} \boldsymbol{W}^{-1} \boldsymbol{\psi}
$$


We substitute this expression in the equation for the constraint itself:

$$
\frac{1}{4 \lambda^{2}} \boldsymbol{\psi}^{\mathrm{T}} \boldsymbol{W}^{-1 \mathrm{~T}} \boldsymbol{W} \boldsymbol{W}^{-1} \boldsymbol{\psi}=\theta^{2} .
$$

This leads to

$$
\lambda=\frac{\sqrt{\boldsymbol{\psi}^{\mathrm{T}} \boldsymbol{W}^{-1} \boldsymbol{\psi}}}{2 \theta} .
$$

This yields the worst earthquake excitation vector

$$
A_{\text {worst }}=\theta \frac{\boldsymbol{W}^{-1} \boldsymbol{\psi}}{\sqrt{\boldsymbol{\psi}^{\mathrm{T}} \boldsymbol{W}^{-1} \boldsymbol{\psi}}} .
$$

The worst response is obtained by substituting Eq. (22) into Eq. (9):

$$
x_{\text {worst }}(t)=\boldsymbol{A}^{\mathrm{T}} \boldsymbol{\psi}=\theta \sqrt{\boldsymbol{\psi}^{\mathrm{T}} \boldsymbol{W}^{-1} \boldsymbol{\psi}}
$$

Once the basic excitation functions $\varphi_{i}(t)$ are chosen, the basic response functions $\psi_{i}(t)$ are readily obtained from Eq. (10). Then, having the information on matrix $\boldsymbol{W}$ and constant $\theta$ from the accelerograms, we find the maximum response by employing Eq. (22).

\section{ELLIPSOIDAL MODELING OF DATA}

\subsection{Basic Ideas}

Consider any collection of data to be processed by the procedure in Section 3. Assume that one record consists of $N$ numerical parameters, so that any observation $j$ of the phenomena is fully described by a point $P_{j}$ in $N$-dimensional Euclidean vector space $E_{N}$.

The problem treated in this section is to find the smallest (in some sense) ellipsoid containing all the observed data, i.e. to set the matrix $W$ in Eq. (14) and the shift of the origin to the center of the ellipsoid.

This problem is rather hard to solve, if the ellipsoid width defining the minimum volume is required; the approach leads to cumbersome optimizations, that may be impractical. So, the problem is treated aiming at a simple, as far as possible, solution. 
In this view, and for simplicity of graphic representation only, let us consider the three-dimensional case (Fig. 1), i.e. a collection of $m$ points $P_{1}, \ldots, P_{m}$ that are the recorded observations of the phenomenon to be included in the ellipsoid and let

$$
\mathbf{x}_{1}=\left(\begin{array}{c}
x_{11} \\
\vdots \\
x_{1 N}
\end{array}\right) ; \quad \mathbf{x}_{2}=\left(\begin{array}{c}
x_{11} \\
\vdots \\
x_{1 N}
\end{array}\right) ; \quad \ldots ; \quad \mathbf{x}_{m}=\left(\begin{array}{c}
x_{m 1} \\
\vdots \\
x_{m N}
\end{array}\right)
$$

be the coordinate column vectors of the points, collecting any relevant parameter to identify the characters of the phenomenon at the observation $P_{i}$, in the reference frame with origin $\mathrm{O}$ and the bases

$$
\left(\mathbf{e}_{1}, \ldots, \mathbf{e}_{N}\right) \quad \text { with } \quad \mathbf{e}_{1}=\left(\begin{array}{l}
1 \\
0 \\
0
\end{array}\right) ; \quad \mathbf{e}_{2}=\left(\begin{array}{l}
0 \\
1 \\
0
\end{array}\right) ; \quad \mathbf{e}_{N=3}=\left(\begin{array}{l}
0 \\
0 \\
1
\end{array}\right) .
$$

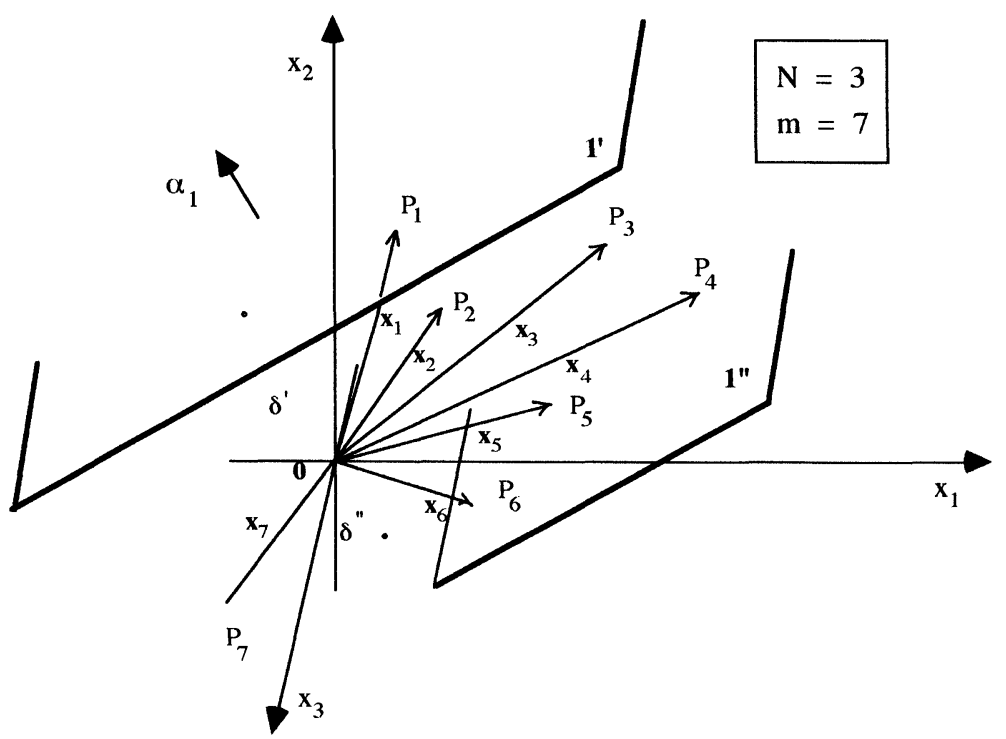

FIGURE 1 
The proposed procedure is conducted basically in two phases:

(A) Find the smallest parallelepiped $\wp$ in $E_{N}$ containing all points $P_{i}$ $(i=1, \ldots, m)$.

(B) Find the smallest ellipsoid in $E_{N}$, also containing all points $P_{i}$ $(i=1, \ldots, m)$, searching in the subset of ellipsoids $\boldsymbol{\varepsilon}_{\lambda}$ that are homothetical to the ellipsoid $\varepsilon_{1}$ inscribed in $\wp$ having the same center as $\wp$ and the principal axes parallel to the sides of $\wp$.

The second phase is straightforward, as it will be shown later. The phase (A) is founded on the solution of two auxiliary problems as follows.

\subsection{Preliminary Statements}

Consider the following auxiliary problems:

Problem $I$ Given the set of $m$ points $P_{1}, \ldots, P_{m}$ in $E_{N}$ consider to find the couple of parallel hyperplanes, say $\mathbf{1}^{\prime}$ and $\mathbf{1}^{\prime \prime}$ (see Fig. 2 for $3 \mathrm{D}$ case), containing all the points and having the minimum distance

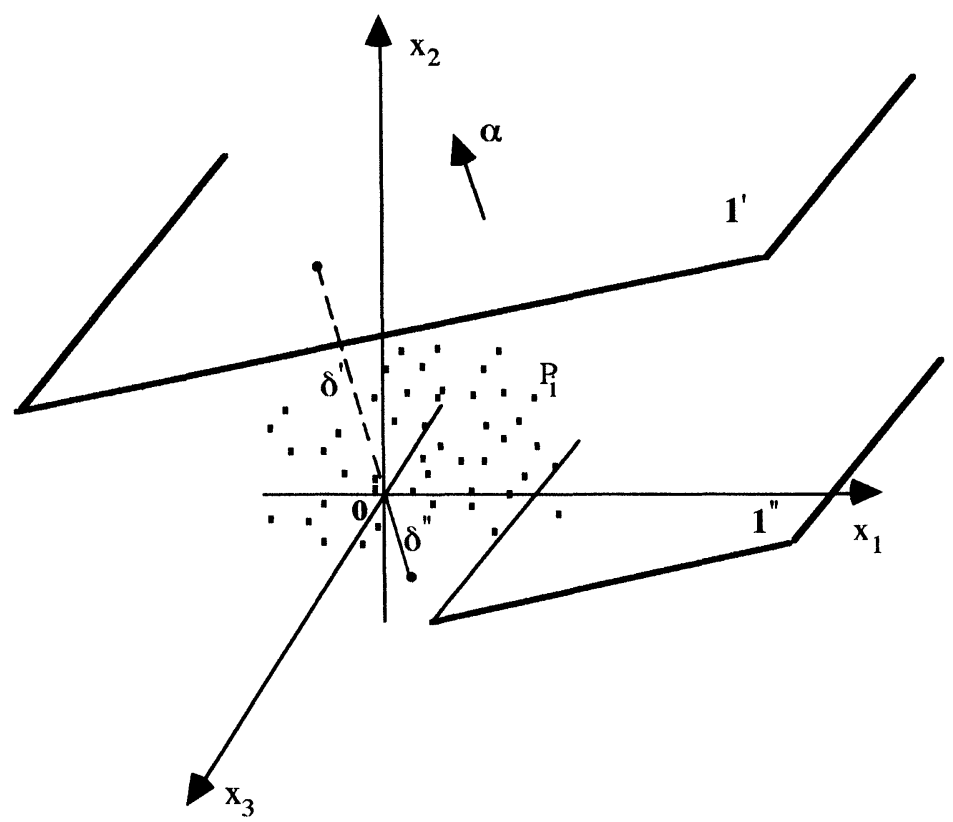

FIGURE 2 
from each other. Let $1^{\prime}$ and $1^{\prime \prime}$ be any two parallel hyperplanes in $E_{N}$ with $\alpha$ being the unit vector orthogonal to both; let $\delta^{\prime}$ and $\delta^{\prime \prime}$ be the orthogonal distance of, respectively, $1^{\prime}$ and $1^{\prime \prime}$ from the point $\mathbf{O}$ assumed to be the origin of the reference frame (see Fig. 2 when $\delta^{\prime}>0$ and $\left.\delta^{\prime \prime}<0\right)$. Any point $P_{i}$ is internal to the strip included by $\pi^{\prime}$ and $\boldsymbol{\pi}^{\prime \prime}$ iff simultaneously

$$
\begin{array}{r}
\boldsymbol{x}_{i}^{\mathrm{T}} \cdot \boldsymbol{\alpha} \leq \delta^{\prime}, \\
\boldsymbol{x}_{i}^{\mathrm{T}} \cdot \boldsymbol{\alpha} \geq \delta^{\prime \prime} .
\end{array}
$$

By applying (26) for $i=1, \ldots, m$, one gets that the strip includes all points iff

$$
\begin{gathered}
X \cdot \boldsymbol{\alpha} \leq \delta^{\prime} \mathbf{1}, \\
\boldsymbol{X} \cdot \boldsymbol{\alpha} \geq \delta^{\prime \prime} \mathbf{1},
\end{gathered}
$$

where

$$
\mathbf{1}=\left(\begin{array}{c}
1 \\
\vdots \\
1
\end{array}\right) \quad \text { and } \quad \boldsymbol{X}=\left(\begin{array}{c}
\boldsymbol{x}_{1}^{\mathrm{T}} \\
\vdots \\
\boldsymbol{x}_{m}^{\mathrm{T}}
\end{array}\right)
$$

The total width of the strip is given by $\delta^{\prime}-\delta^{\prime \prime}$. Therefore, the first problem turns into:

$$
\text { Find } \alpha \in S_{N 1}: \quad\left\{\begin{array}{l}
\boldsymbol{X} \cdot \boldsymbol{\alpha} \leq \delta^{\prime} \mathbf{1} \\
\boldsymbol{X} \cdot \boldsymbol{\alpha} \geq \delta^{\prime \prime} \mathbf{1} \\
\delta^{\prime}-\delta^{\prime \prime}=\min
\end{array}\right.
$$

with $S_{N 1}$ being the unit sphere in $E_{N}$.

This problem is approached in two steps:

Step I.1 For any given $\alpha \in S_{N 1}$

$$
\text { Find }\left(\delta^{\prime \alpha}, \delta^{\prime \prime \alpha}\right) \in R^{2}:\left\{\begin{array}{l}
\boldsymbol{X} \cdot \boldsymbol{\alpha} \leq \delta^{\prime} \mathbf{1}, \\
\boldsymbol{X} \cdot \boldsymbol{\alpha} \geq \delta^{\prime \prime} 1 \\
\delta^{\prime \alpha}-\delta^{\prime \prime \alpha}=\min
\end{array}\right.
$$


It is trivially proved that

$$
\delta^{\prime \alpha}=\max _{i} \boldsymbol{x}_{i}^{\mathrm{T}} \cdot \boldsymbol{\alpha}=\boldsymbol{x}_{r}^{\mathrm{T}} \cdot \boldsymbol{\alpha} ; \quad \delta^{\prime \prime \alpha}=\min _{i} \boldsymbol{x}_{i}^{\mathrm{T}} \cdot \boldsymbol{\alpha}=\boldsymbol{x}_{s}^{\mathrm{T}} \cdot \boldsymbol{\alpha} .
$$

Step I.2 In order to find the smallest strip containing all points, consider the following problem:

$$
\text { Find } \boldsymbol{\alpha}_{1} \in R^{N}: \quad\left\{\begin{array}{l}
\boldsymbol{X} \cdot \boldsymbol{\alpha}_{1} \leq \delta^{\prime \alpha_{1}} \mathbf{1} \\
\boldsymbol{X} \cdot \boldsymbol{\alpha}_{1} \geq \delta^{\prime \prime \alpha_{1}} \mathbf{1} \\
\boldsymbol{\alpha}_{1}^{\mathrm{T}} \cdot \boldsymbol{\alpha}_{1}=\mathbf{1} \\
\delta^{\prime \alpha_{1}}-\delta^{\prime \prime \alpha_{1}}=\min
\end{array}\right.
$$

where $\delta^{\prime \alpha_{1}}$ and $\delta^{\prime \prime \alpha_{1}}$ are intrinsically related to $\alpha_{1}$ by Eq. (31).

The above problem is a non-linear optimization problem, mainly because of the constraint $\boldsymbol{\alpha}_{1}^{\mathrm{T}} \boldsymbol{\alpha}_{1}=1$.

Any constrained search procedure can be applied to find the optimal vector $\boldsymbol{\alpha}_{1}$.

The simplest, and also effective, as tested in this investigation, is a random walk search. The only difficulty lies in generating unit random vectors $\boldsymbol{\alpha}$ 's checking the condition (32c).

The following procedure is suggested. Let $\beta$ be a generic unit random vector and $\alpha$ the unit vector satisfying the condition (32d). Let $n_{i}$ and $s_{i}(i=1, \ldots, N)$ be any random numbers in $[0,1], \vartheta_{i}=2 \pi n_{i}$ is the random angle in $[0,2 \pi]$ of $\boldsymbol{\beta}$ and $\mathbf{S G}_{i}$ the sign of the $i$ th component of $\boldsymbol{\beta}$ with

$$
\begin{cases}\mathrm{SG}_{i}=+1 & \text { if } s_{i} \leq 0.5 \\ \mathrm{SG}_{i}=-1 & \text { if } s_{i}>0.5\end{cases}
$$

The components of $\boldsymbol{\beta}$ are chosen through the following steps:

(a) $R_{N}=1$,

(b) $R_{N-i}=\sin ^{2}\left(\vartheta_{i}\right) * R_{N-i+1}(i=1, \ldots, N-1)$ and $\vartheta_{i}=2 \pi n_{i}$,

(c) $\beta_{i+1}=\mathrm{SG}_{i+1} * \sqrt{\left(R_{i+1}-R_{i}\right)}(i=N-1, \ldots, 1)$.

Problem I is thus solved after Steps I.1, II.2.

Problem II Let $\boldsymbol{\alpha}_{1}$ be found, and the first minimal strip obtained, with width $\delta_{1}^{\prime}-\delta_{1}^{\prime \prime}=d_{1}$. 
Let $\boldsymbol{\alpha}_{1}, \ldots, \boldsymbol{\alpha}_{k}(k \leq N)$ be given unit vectors, mutually orthogonal (i.e. $\boldsymbol{\alpha}_{i}^{\mathrm{T}} \cdot \boldsymbol{\alpha}_{j}=\delta_{i j}$ ), and consider the following Problem (II):

$$
\text { Find } \boldsymbol{\alpha}_{k+1}: \quad\left\{\begin{array}{l}
\boldsymbol{X} \cdot \boldsymbol{\alpha}_{k+1} \leq \delta^{\prime \alpha_{k+1}} \mathbf{1} \\
\boldsymbol{X} \cdot \boldsymbol{\alpha}_{k+1} \geq \delta^{\prime \prime \alpha_{k+1}} \mathbf{1} \\
\boldsymbol{\alpha}_{k+1}^{\mathrm{T}} \boldsymbol{\alpha}_{k+1}=\mathbf{1} \\
\boldsymbol{\alpha}_{i}^{\mathrm{T}} \boldsymbol{\alpha}_{k+1}=\mathbf{0} \quad(i=1, \ldots, k) \\
\delta^{\prime \alpha_{k+1}}-\delta^{\prime \prime \alpha_{k+1}}=\text { min. }
\end{array}\right.
$$

Problem II exhibits a number, say $k$, of constraints in addition to Problem I and it can be viewed at as a generalization of Problem I, by making reference to $\Im_{N-k}$, the vector subspace of $E_{N}$ orthogonal to $\alpha_{1}, \ldots, \alpha_{k}$.

To generate $\Im_{N-k}$ one has to infer an orthogonal unit minimal base, say $\left(\boldsymbol{\beta}_{1}^{k+1}, \ldots, \boldsymbol{\beta}_{N-k}^{k+1}\right)$, of $\Im_{N-k}$. To this aim one can take recourse to the Schmidt's orthogonalization procedure, as follows.

Let $\boldsymbol{\beta}_{1}^{k}, \ldots, \boldsymbol{\beta}_{N-k+1}^{k}$ be the minimal orthogonal base of $\Im_{N-k+1}$ so that both sets of unit vectors,

$$
B^{k}=\left(\begin{array}{c}
\boldsymbol{\alpha}_{1}^{\mathrm{T}} \\
\vdots \\
\boldsymbol{\alpha}_{k-1}^{\mathrm{T}} \\
\left(\boldsymbol{\beta}_{1}^{k}\right)^{\mathrm{T}} \\
\left(\boldsymbol{\beta}_{2}^{k}\right)^{\mathrm{T}} \\
\vdots \\
\left(\boldsymbol{\beta}_{N-k+1}^{k}\right)^{\mathrm{T}}
\end{array}\right), \quad B^{k+1}=\left(\begin{array}{c}
\boldsymbol{\alpha}_{1}^{\mathrm{T}} \\
\vdots \\
\boldsymbol{\alpha}_{k-1}^{\mathrm{T}} \\
\boldsymbol{\alpha}_{k}^{\mathrm{T}} \\
\left(\boldsymbol{\beta}_{1}^{k+1}\right)^{\mathrm{T}} \\
\vdots \\
\left(\boldsymbol{\beta}_{N-k}^{k+1}\right)^{\mathrm{T}}
\end{array}\right),
$$

are orthogonal unit bases for the entire $E_{N}$, the first is a known base, the second is known up to $\boldsymbol{\alpha}_{k}$.

Note that

$$
B^{1}=\left(\begin{array}{c}
\mathbf{e}_{1} \\
\mathbf{M} \\
\mathbf{e}_{N}
\end{array}\right) .
$$


One has to infer $B^{k+1}$ from $B^{k}$. Assuming $\boldsymbol{\gamma}_{0}=\boldsymbol{\alpha}_{k}$ and following Schmidt's method one sets:

$$
\begin{aligned}
& \boldsymbol{\gamma}_{1}=\lambda_{1}^{1} \boldsymbol{\alpha}_{k}+\boldsymbol{\beta}_{2}^{k}, \\
& \boldsymbol{\gamma}_{2}=\lambda_{2}^{1} \boldsymbol{\alpha}_{k}+\lambda_{2}^{2} \boldsymbol{\gamma}_{1}+\boldsymbol{\beta}_{3}^{k}, \\
& \boldsymbol{\gamma}_{3}=\lambda_{3}^{1} \boldsymbol{\alpha}_{k}+\lambda_{3}^{2} \boldsymbol{\gamma}_{1}+\lambda_{3}^{3} \boldsymbol{\gamma}_{2}+\boldsymbol{\beta}_{4}^{k}, \\
& \vdots \\
& \boldsymbol{\gamma}_{n-k}=\lambda_{n-k}^{1} \boldsymbol{\alpha}_{k}+\lambda_{n-k}^{2} \boldsymbol{\gamma}_{1}+\lambda_{N-k}^{3} \boldsymbol{\gamma}_{2}+\cdots+\boldsymbol{\beta}_{N-k+1}^{k},
\end{aligned}
$$

where, by orthogonalization

$$
\begin{array}{ll}
\lambda_{1}^{1}=-\left(\boldsymbol{\beta}_{2}^{k}\right)^{\mathrm{T}} \boldsymbol{\alpha}_{k} ; & \lambda_{2}^{2}=-\frac{\left(\boldsymbol{\beta}_{3}^{k}\right)^{\mathrm{T}} \boldsymbol{\gamma}_{1}}{\left(\boldsymbol{\gamma}_{1}\right)^{\mathrm{T}} \boldsymbol{\gamma}_{1}} \\
\lambda_{2}^{1}=-\left(\boldsymbol{\beta}_{3}^{k}\right)^{\mathrm{T}} \boldsymbol{\alpha}_{k}, & \lambda_{3}^{2}=-\frac{\left(\boldsymbol{\beta}_{4}^{k}\right)^{\mathrm{T}} \boldsymbol{\gamma}_{1}}{\left(\boldsymbol{\gamma}_{1}\right)^{\mathrm{T}} \boldsymbol{\gamma}_{1}} \quad \lambda_{3}^{3}=-\frac{\left(\boldsymbol{\beta}_{4}^{k}\right)^{\mathrm{T}} \boldsymbol{\gamma}_{2}}{\left(\boldsymbol{\gamma}_{2}\right)^{\mathrm{T}} \boldsymbol{\gamma}_{2}} \\
\begin{array}{l}
\lambda_{3}^{1}=-\left(\boldsymbol{\beta}_{4}^{k}\right)^{\mathrm{T}} \boldsymbol{\alpha}_{k}, \\
\vdots
\end{array} & \vdots \\
\lambda_{N-k}^{1}=-\left(\boldsymbol{\beta}_{N-k+1}^{k}\right)^{\mathrm{T}} \boldsymbol{\alpha}_{k}, & \lambda_{N-k}^{2}=-\frac{\left(\boldsymbol{\beta}_{N-k+1}^{k}\right)^{\mathrm{T}} \boldsymbol{\gamma}_{1}}{\left(\boldsymbol{\gamma}_{1}\right)^{\mathrm{T}} \boldsymbol{\gamma}_{1}}, \lambda_{N-k}^{3}=-\frac{\left(\boldsymbol{\beta}_{N-k+1}^{k}\right)^{\mathrm{T}} \boldsymbol{\gamma}_{2}}{\left(\boldsymbol{\gamma}_{2}\right)^{\mathrm{T}} \boldsymbol{\gamma}_{2}}, \\
\ldots, \lambda_{N-k}^{N-k}=-\frac{\left(\boldsymbol{\beta}_{N-k+1}^{k}\right)^{\mathrm{T}} \boldsymbol{\gamma}_{N-k-1}}{\left(\boldsymbol{\gamma}_{N-k-1}\right)^{\mathrm{T}} \boldsymbol{\gamma}_{N-k-1}}
\end{array}
$$

After the orthogonal vectors $\boldsymbol{\gamma}_{i}$ have been found, one can normalize:

$$
\boldsymbol{\beta}_{i}^{k+1}=\frac{\boldsymbol{\gamma}_{i}}{\left|\boldsymbol{\gamma}_{i}\right|}, \quad i=1, \ldots, N-k
$$

Note that each $\boldsymbol{\gamma}_{i}$, for $i>0$, and consequently each $\boldsymbol{\beta}_{i}^{k+1}$ is orthogonal to $\boldsymbol{\alpha}_{j}, \forall j=1, \ldots, k$. In fact, every $\boldsymbol{\gamma}_{i}$ turns out to be a linear combination of vector $\boldsymbol{\alpha}_{k}$ and vectors $\boldsymbol{\beta}_{i}^{k}$. All these $\boldsymbol{\beta}$ vectors are assumed to have been built up orthogonal to $\boldsymbol{\alpha}_{1}, \ldots, \boldsymbol{\alpha}_{k}$ in the 
previous step. Whence one can write, for suitable $a_{i j}$,

$$
\boldsymbol{\gamma}_{i}=a_{i 0} \boldsymbol{\alpha}_{k}+\sum_{r=1}^{N-k+1} a_{i r} \boldsymbol{\beta}_{r}^{k}
$$

and

$$
\boldsymbol{\gamma}_{i} \cdot \boldsymbol{\alpha}_{j}=a_{i 0} \boldsymbol{\alpha}_{k} \cdot \boldsymbol{\alpha}_{j}+\sum_{r=1}^{N-k+1} a_{i r} \boldsymbol{\beta}_{r}^{k} \cdot \boldsymbol{\alpha}_{j}=0
$$

Therefore, problem (34) is reduced to:

$$
\text { Find } \boldsymbol{\alpha}_{k+1} \in \Im_{N-k}: \quad\left\{\begin{array}{l}
X \boldsymbol{\alpha}_{k+1} \leq \delta^{\prime \alpha_{k+1}} \mathbf{1}, \\
\boldsymbol{X} \boldsymbol{\alpha}_{k+1} \geq \delta^{\prime \prime \alpha_{k+1}} \mathbf{1} \\
\boldsymbol{\alpha}_{k+1}^{\mathrm{T}} \boldsymbol{\alpha}_{k+1}=\mathbf{1} \\
\delta^{\prime \alpha_{k+1}}-\delta^{\prime \prime \alpha_{k+1}}=\text { min }
\end{array}\right.
$$

where $\boldsymbol{\alpha}_{k+1} \in \Im_{N-k}$ means

$$
\boldsymbol{\alpha}_{k+1}=\sum_{i=1}^{N-K} \eta_{i}^{k} \boldsymbol{\beta}_{i}^{k+1},
$$

with

$$
\left(\boldsymbol{\alpha}_{k+1}\right)^{\mathrm{T}} \boldsymbol{\alpha}_{k+1}=\sum_{i=1}^{N-k} \sum_{r=1}^{N-k} \eta_{i}^{k} \eta_{r}^{k}\left(\boldsymbol{\beta}_{i}^{k+1}\right)^{\mathrm{T}} \boldsymbol{\beta}_{i}^{k+1}
$$

whence, by orthonormality of $\boldsymbol{\beta}$ 's,

$$
\left(\boldsymbol{\alpha}_{k+1}\right)^{\mathrm{T}} \boldsymbol{\alpha}_{k+1}=\sum_{i=1}^{N-k}\left(\eta_{i}^{k}\right)^{2} .
$$

With the above relations, Problem II turns to the following one:

$$
\text { Find } \boldsymbol{\eta}^{k} \in \Im_{N-k}: \quad\left\{\begin{array}{l}
\boldsymbol{\alpha}_{k+1}=\sum_{i=1}^{N-K} \eta_{i}^{k} \boldsymbol{\beta}_{i}^{k+1} \\
\boldsymbol{X} \boldsymbol{\alpha}_{k+1} \leq \delta^{\prime \alpha_{k+1}} \mathbf{1} \\
\boldsymbol{X} \boldsymbol{\alpha}_{k+1} \geq \delta^{\prime \prime \alpha_{k+1}} \mathbf{1} \\
\left(\boldsymbol{\eta}^{k}\right)^{\mathrm{T}} \boldsymbol{\eta}^{k}=\mathbf{1} \\
\delta^{\prime \alpha_{k+1}}-\delta^{\prime \prime \alpha_{k+1}}=\min
\end{array}\right.
$$


Thus, one recognizes that Problem II is reduced to Problem I in the vector space $E_{N-k}$.

\subsection{Search of the Smallest Parallelepiped $\wp$ in $E_{N}$ Containing the Data Points}

This part of the paper represents the phase A of the main problem, and it is articulated in $N$ steps.

Step A.1 Solve Problem I, find $\alpha_{1}, \delta^{\prime \alpha_{1}}, \delta^{\prime \prime \alpha_{1}}$.

After this step a first strip between hyperplanes $\mathbf{1}^{\prime}$ and $\mathbf{1}^{\prime \prime}$ is determined.

Step A.2 Solve Problem II, with $k=1$, in the form (46) and find $\boldsymbol{\alpha}_{k+1}, \delta^{\prime \alpha_{k-1}}, \delta^{\prime \prime \alpha_{k+1}}$.

Step A.3, $\ldots, A . N$ Iterate step A.2 for $k=2, \ldots, N$. After step A. $N, N$ couple of hyperplanes are individuated, each couple defines a minimal strip in $E_{N}$ containing all $m$ points.

By iterating these $N$ steps, the minimal parallelepiped $\wp$ is found.

\subsection{Search the "Smallest" Ellipsoid Containing all Points}

The "smallest" ellipsoid is assumed to have the same centroid as $\wp$ and principal axis parallel to the edges of $\wp$ which are identified in the unit vectors $\boldsymbol{\alpha}_{1}, \ldots, \boldsymbol{\alpha}_{N}$. The diametral lengths are assumed proportional, respectively, to $d_{1}=\delta^{\prime \alpha_{1}}-\delta^{\prime \prime \alpha_{1}}, \ldots, d_{N}=\delta^{\prime \alpha_{N}}-\delta^{\prime \prime \alpha_{N}}$.

Let $x_{0}$ be the position vector of the centroid of $\wp$, and shift the origin of the reference system to $x_{0}$. Thus the data points are identified in the new reference frame by

$$
\boldsymbol{A}_{i}=\boldsymbol{x}_{i}-\boldsymbol{x}_{0}, \quad i=1, \ldots, m,
$$

so that the ellipsoid is identified by

$$
\boldsymbol{A}^{\mathrm{T}} \boldsymbol{W} \boldsymbol{A} \leq \theta^{2} .
$$

In order to identify matrix $\boldsymbol{W}$, let $\boldsymbol{R}$ be the rotation matrix yielding

$$
\boldsymbol{R} \boldsymbol{B}_{1}=\left(\begin{array}{c}
\alpha_{i}^{\mathrm{T}} \\
\vdots \\
\alpha_{N}^{\mathrm{T}}
\end{array}\right)=B_{N}
$$


In the frame of reference $B_{N}$, the equation of the ellipsoid is given by

$$
\sum_{i=1}^{m} \frac{z_{i}^{2}}{d_{i}^{2}}=Z^{\mathrm{T}} \boldsymbol{Q Z} \leq \theta^{2}
$$

where $z_{i}$ are the coordinates of any point with respect of $B_{N}$ and

$$
\boldsymbol{Q}=\left[\begin{array}{ccccc}
1 / d_{1}^{2} & 0 & . & . & 0 \\
0 & \cdot & . & . & \cdot \\
\cdot & 0 & 1 / d_{i}^{2} & 0 & \\
. & . & \cdot & \cdot & 0 \\
0 & \cdot & \cdot & 0 & 1 / d_{N}^{2}
\end{array}\right]
$$

In the reference frame $B_{1}$ one has the coordinates $A$ of any point $P$ related to the corresponding coordinates in $B_{N}$

$$
\boldsymbol{P}=B_{1}^{\mathrm{T}} \boldsymbol{A}=B_{N}^{\mathrm{T}} \boldsymbol{Z}=\boldsymbol{B}_{1}^{\mathrm{T}} \boldsymbol{R}^{\mathrm{T}} \boldsymbol{Z}
$$

whence

$$
\boldsymbol{A}=\boldsymbol{R}^{\mathrm{T}} \boldsymbol{Z} \text { and } \boldsymbol{Z}=\boldsymbol{R} \boldsymbol{A} .
$$

Therefore the equation of the ellipsoid is

$$
\boldsymbol{Z}^{\mathrm{T}} \boldsymbol{Q Z}=\boldsymbol{A}^{\mathrm{T}} \boldsymbol{R}^{\mathrm{T}} \boldsymbol{Q R} \boldsymbol{A} \leq \theta^{2}
$$

whence, in $B_{1}$ (having again shifted back to $x_{0}$ )

$$
\boldsymbol{A}^{\mathrm{T}} \boldsymbol{W} \boldsymbol{A} \leq \theta^{2}
$$

where $\boldsymbol{W}=\boldsymbol{R}^{\mathrm{T}} \boldsymbol{Q R}$.

The above considerations produce the matrix $\boldsymbol{W}$.

Now the minimal value for $\theta^{2}$ can be searched by unidimensional search

$$
\theta^{2}=\max _{i=1, \ldots, m} \boldsymbol{A}_{i}^{\mathrm{T}} \boldsymbol{W} \boldsymbol{A}_{i}
$$


Note that the above "minimal" ellipsoid may not be the absolute minimum, since it is minimal under the assumption that it is related to the minimal parallelepiped.

The ellipsoid so found is in general included between the ellipsoid contained in $\wp$ and the one containing $\wp$.

\subsection{Numerical Application}

A numerical application of the procedure proposed above has been performed; in order to show the results graphically, the example has been carried out for $N=3$.

Let us consider a set of points $P_{m}(m=14)$, the coordinates are given in Table I. The unit vectors $\boldsymbol{\alpha}_{1}, \boldsymbol{\alpha}_{2}, \boldsymbol{\alpha}_{3}$, in $E_{3}$, orthogonal to the couples of planes that define the minimal strips containing all points $P$, are

$$
\boldsymbol{\alpha}_{1}=\left(\begin{array}{c}
0.2052 \\
-0.670 \\
0.7130
\end{array}\right) ; \quad \boldsymbol{\alpha}_{2}=\left(\begin{array}{c}
-0.824 \\
-0.510 \\
-0.242
\end{array}\right) ; \quad \boldsymbol{\alpha}_{3}=\left(\begin{array}{c}
0.5269 \\
-0.538 \\
-0.657
\end{array}\right)
$$

The minimal distances between the planes are

$$
d_{1}=647 ; \quad d_{2}=981 ; \quad d_{3}=3240 .
$$

Figure 3 shows the minimal parallelepiped $\wp$, the ellipsoid $\varepsilon_{1}$ contained in $\wp$ and the principal planes of the ellipsoids.

Figure 4 shows the sectional view of the three principal planes.

Figure 5 shows the ellipsoid $\boldsymbol{\varepsilon}_{\lambda}$ found by enlarging homothetically $\varepsilon_{1}$ in order to include all points.

TABLE I

\begin{tabular}{rrrrrrrrrrrrrrr}
\hline & $P_{1}$ & $P_{2}$ & $P_{3}$ & $P_{4}$ & $P_{5}$ & $P_{6}$ & $P_{7}$ & $P_{8}$ & $P_{9}$ & $P_{10}$ & $P_{11}$ & $P_{12}$ & $P_{13}$ & $P_{14}$ \\
\hline$x_{1}$ & 100 & -300 & 700 & 400 & -200 & 400 & 0 & 100 & -300 & 700 & 400 & -200 & -750 & 1380 \\
$x_{2}$ & 500 & 550 & 450 & 470 & 500 & 500 & 500 & -200 & 500 & 800 & 600 & 800 & 1370 & -250 \\
$x_{3}$ & 800 & 800 & 600 & 600 & 400 & 400 & 200 & 200 & 100 & 100 & 900 & 900 & 1600 & -260 \\
\hline
\end{tabular}




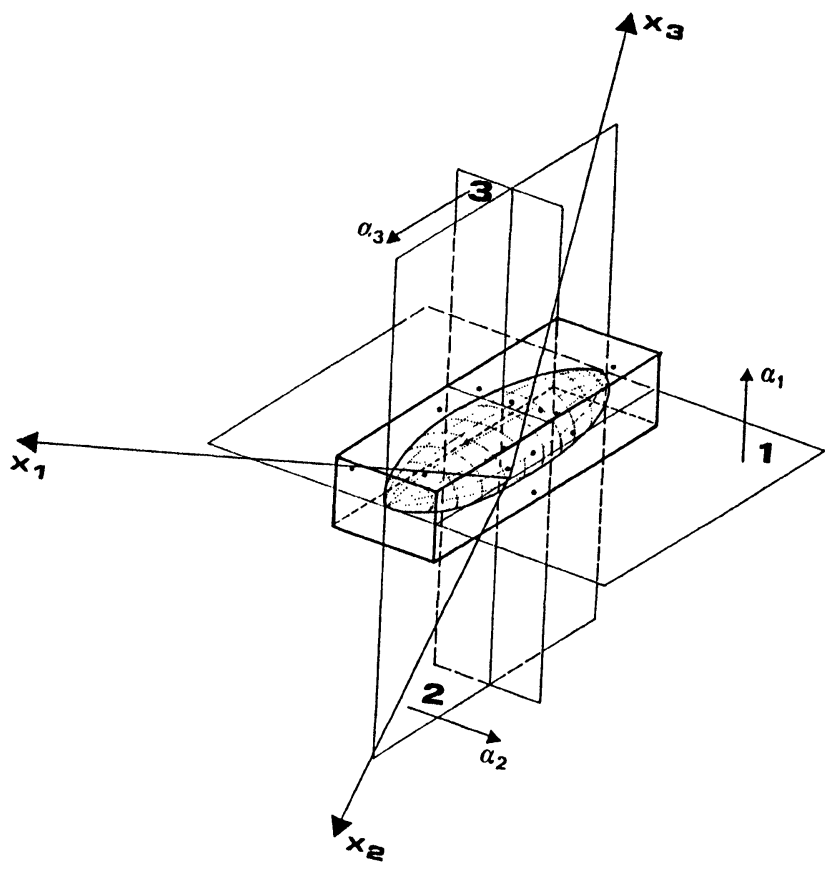

FIGURE 3

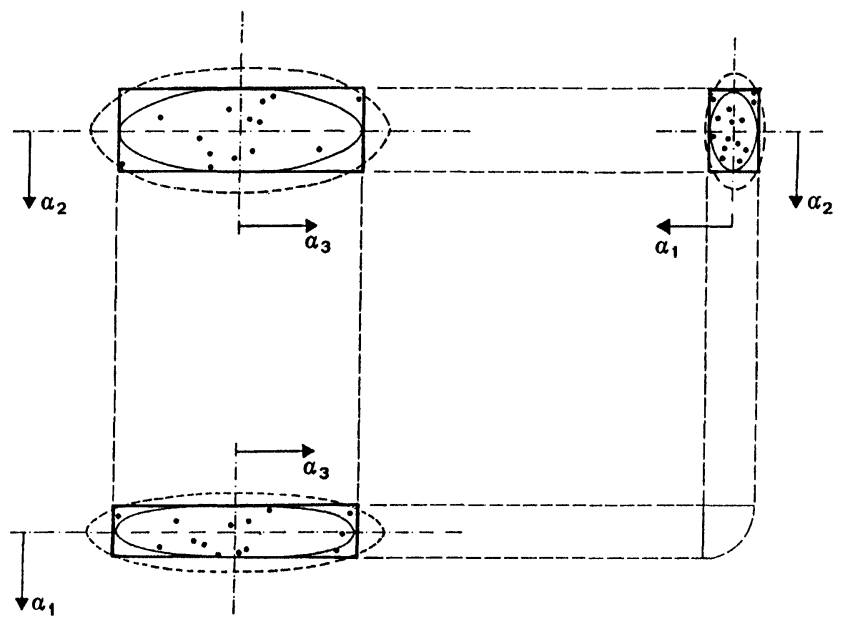

FIGURE 4 


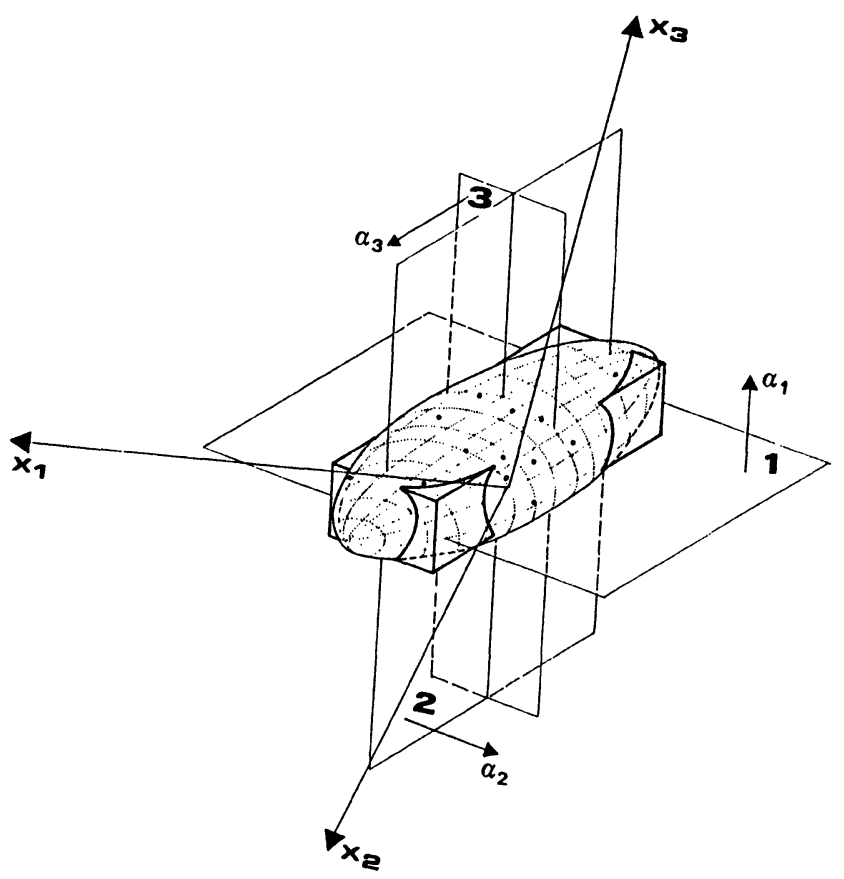

FIGURE 5

\section{Acknowledgements}

The work had been initiated in the Fall Semester of the academic year 1992-93 when A. Baratta was a Visiting Research Professor and G. Zuccaro a Visiting Research Assistant Professor at the Department of Mechanical Engineering, Florida Atlantic University. The work by I. Elishakoff has been supported by the National Center for Earthquake Engineering Research through two grants. This support is sincerely appreciated.

\section{References}

[1] R.F. Drenick, Model-free design of aseismic structures, Journal of Engineering Mechanics Division, 96 (1970) 483-493.

[2] R.F. Drenick, On the class of the non-robust problems in stochastic dynamics, in Stochastic Problems in Dynamics (B.L. Clarkson, Ed.), Pitman, London, 237-255, 1977. 
[3] R.F. Drenick, F. Novomestky and G. Bagchi, Critical excitation of structures, in Wind and Seismic Effects, Proceedings of the 12th Joint UJNR Panel Conference, US National Bureau of Standards Special Publication, 133-142, 1984.

[4] R.F. Drenick and C.B. Yun, Reliability of seismic resistance predictions, Journal of the Structural Division, 105 (1979) 1879-1891.

[5] M. Shinozuka, Maximum structural response to seismic excitations, Journal of Engineering Mechanics Division, 96 (1970) 729-738.

[6] I. Elishakoff and B. Pletner, Analysis of base excitation as an uncertain function with specified bounds on it and its derivatives, in Structural Vibration and Acoustics (T.C. Huang et al., Eds.), DE-Vol. 34, ASME (1991) 177-184.

[7] R.F. Drenick, Personal communications to I. Elishakoff, 1989-1992.

[8] A. Baratta, Earthquake simulation in aseismic engineering, Proc. Int. Symp. Simulation of Systems, Sorrento, Italy, North-Holland, 1979, pp. 517-536.

[9] A. Baratta and G. Zuccaro, High-reliability aseismic design, Proc. Tenth World Conference on Earthquake Engineering, Madrid, 1992.

[10] F.C. Schweppe, Uncertain Dynamical Systems, Prentice Hall, Englewood Cliffs, NJ, 1973.

[11] Y. Ben-Haim and I. Elishakoff, Convex Models of Uncertainty in Applied Mechanics, Elsevier Science Publishers, Amsterdam, 1990.

[12] I. Elishakoff and Y. Ben-Haim, Dynamics of a thin cylindrical shell under impact with limited deterministic information on its initial imperfection, Structural Safety, 8 (1990) 103-112.

[13] Y. Ben-Haim, G. Chen and T.T. Soong, Maximum structural response using convex models, Journal of Engineering Mechanics, 122 (1996) 325-334.

[14] I. Elishakoff, Essay on probability, convexity and earthquake engineering, Proceedings of the 5th National Italian Conference on Earthquake Engineering, Palermo, 1991.

[15] P.J. Kelly and M.L. Weiss, Geometry and Convexity, John Wiley \& Sons, New York, 1979.

[16] A. Baratta, Analisi dei dati e simulazione per la sicurezza antisismica delle costruzioni, Proc. Ingegneria Sismica in Italia, Udine, Italy, 1980.

[17] A. Baratta, Optimized earthquake simulation for high-reliability aseismic design, Proc, 3rd Int. Seminar "Reliability of Nuclear Power Plant", Paris, France, 1981.

[18] G. Zuccaro, Accelerogramma di progetto per verifiche ad alta affidabilita', analisi costi-benefici. Proc. 5th National Congress L'ingegneria Sismica in Italia, Palermo, Italy, 1991.

[19] I. Elishakoff, R.T. Haftka and J.J. Fang, Structural design under bounded uncertainity - Optimization with anti-optimization, Computers and Structures, 53 (1994) 1401-1405.

[20] I. Elishakoff, An idea of the uncertainty triangle, The Shock and Vibration Digest, Oct. 1991, p. 1 (Editorial). 


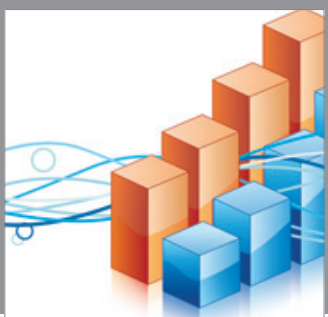

Advances in

Operations Research

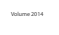

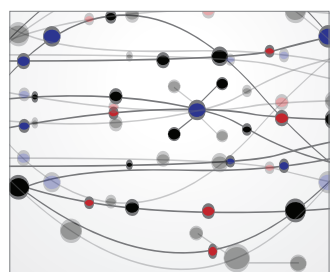

\section{The Scientific} World Journal
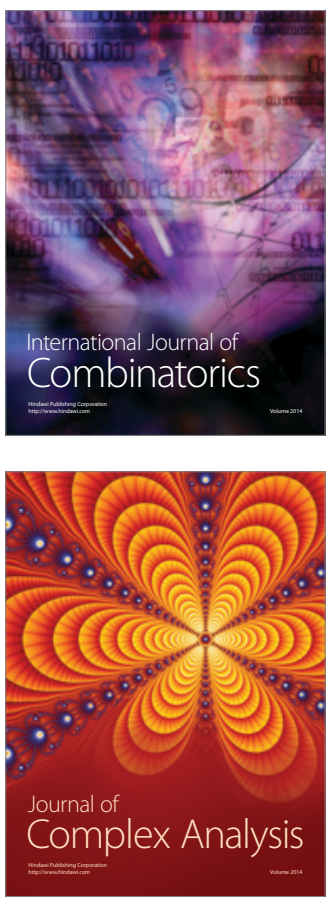

International Journal of

Mathematics and

Mathematical

Sciences
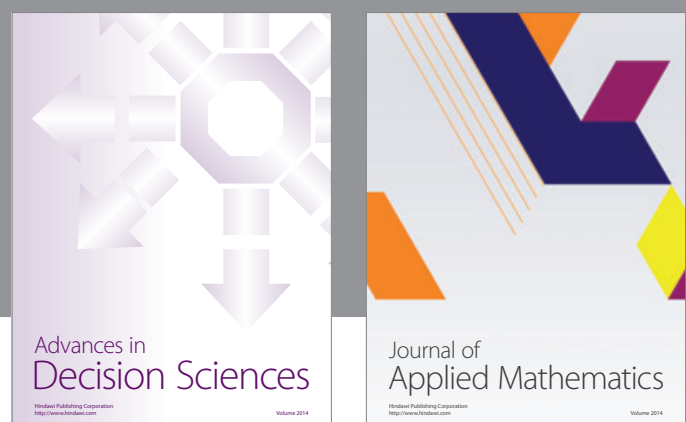

Journal of

Applied Mathematics
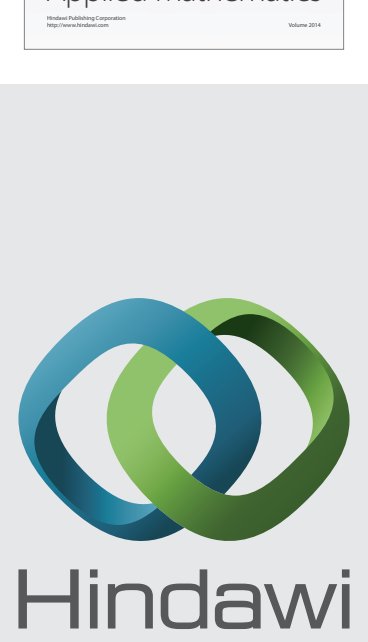

Submit your manuscripts at http://www.hindawi.com
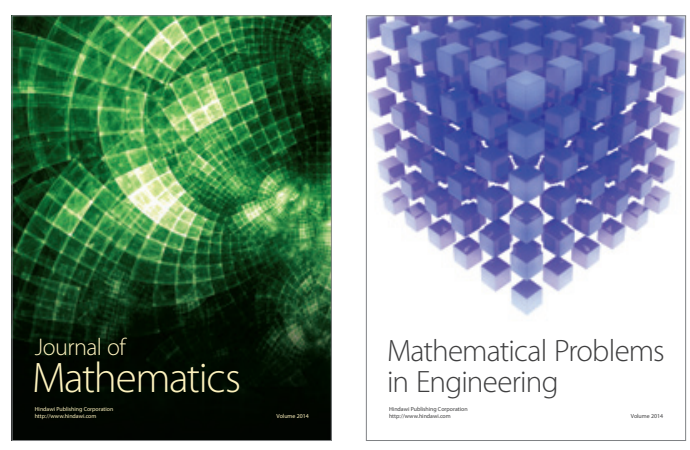

Mathematical Problems in Engineering
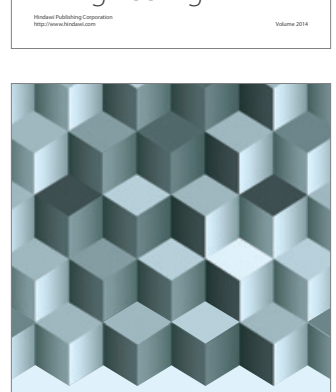

Journal of

Function Spaces
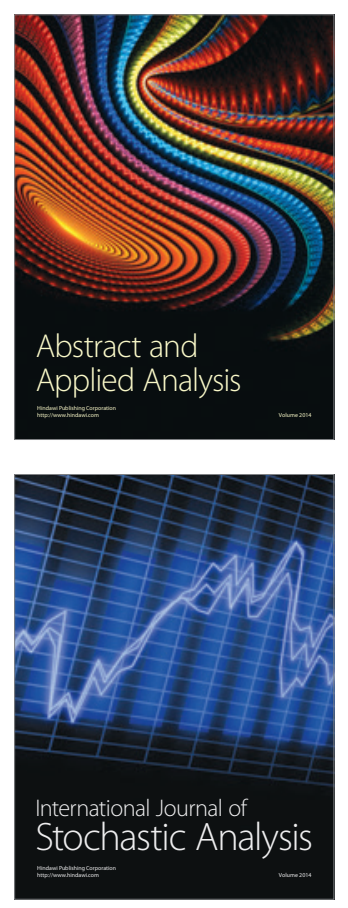

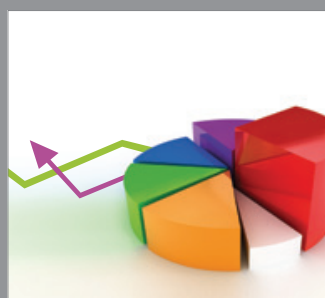

ournal of

Probability and Statistics

Promensencen
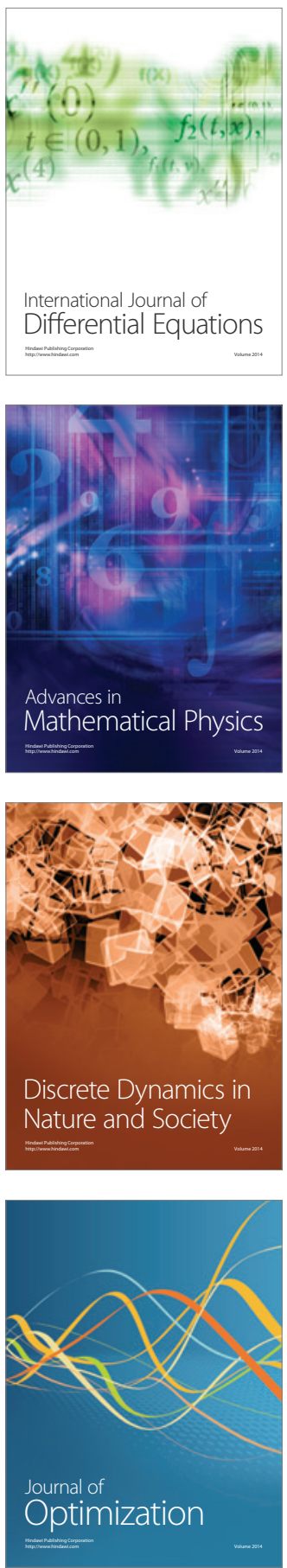